\title{
Perfil lipoprotéico de cadelas submetidas à ovário-histerectomia com e sem reposição estrogênica
}

[Lipoproteic profile of bitches submitted to ovaryhysterectomy with and without estrogen administration]

\author{
C. Schmidt ${ }^{1}$, M.D. Lopes $^{2}$, M.C. Silva ${ }^{3}$, R.A. Fighera ${ }^{3}$, T.M. Souza ${ }^{3}$ \\ ${ }^{1}$ Departamento de Clínica de Pequenos Animais da UFSM \\ Hospital Veterinário - Faixa de Camobi, km 9 \\ Campus Universitário \\ 97105-900 - Santa Maria, RS \\ ${ }^{2}$ Departamento de Radiologia e Reprodução Veterinária da FMVZ - UNESP -Botucatu, SP \\ ${ }^{3}$ Médico Veterinário. Bolsista do CNPq. Seção de Patologia Veterinária da UFSM - Santa Maria, RS
}

\section{RESUMO}

Doze cadelas mestiças, adultas, foram submetidas à ovário-histerectomia e posteriormente distribuídas em dois grupos de seis animais. Um dos grupos recebeu estrógenos naturais conjugados na dose de $0,01 \mathrm{mg} / \mathrm{kg}$, via oral, a cada 48 horas, durante 12 meses, e o outro serviu como controle. A cada 60 dias foram realizadas colheitas de sangue e obtidos os valores de colesterol total, triglicerídeos, HDL, LDL e VLDL. Houve elevação do colesterol total e do LDL nas fêmeas sem reposição de hormônio. No grupo que recebeu estrógenos houve elevação do HDL. A ovário-histerectomia influenciou significativamente o perfil lipoprotéico; a reposição com estrógenos foi capaz de preservar esse perfil nas cadelas castradas.

Palavras-chave: cão, ovário-histerectomia, colesterol, lipoproteínas, estrógeno

\begin{abstract}
Twelve mixed-bred, bitches were divided in two groups of six animals each after ovariohysterectomy. One group received $0.01 \mathrm{mg} / \mathrm{kg}$ per os of natural conjugated estrogens every $48 \mathrm{~h}$ for 12 months; the other group, used as control, was not treated with hormones. Blood samples were collected every 60 days and the levels of total cholesterol, triglycerides, HDL, LDL and VLDL were determined. Ovariohysterectomy affected the lipoproteic profile, increasing the total cholesterol and LDL levels; it also showed that the estrogen supplementation maintained the lipoproteic profile of castrated bitches within normal levels.
\end{abstract}

Keywords: bitch, ovariohysterectomy, cholesterol, lipoprotein, estrogen

\section{INTRODUÇÃO}

Em mulheres, as frações lipídicas apresentam modificações importantes após a menopausa, habitualmente com brusca elevação na colesterolemia total e na fração LDL, com discreta e progressiva diminuição da HDL, elevando o risco de eventos coronários (Giannini, 2001).
$\mathrm{Na}$ espécie canina, as alterações lipoprotéicas relacionadas com a diminuição dos hormônios ovarianos só foi relatada durante a variação fisiológica do ciclo estral, quando houve aumento na concentração plasmáticas de colesterol no metaestro e aumento da fração LDL com diminuição da HDL no pró-estro (Downs et al., 1994).

Recebido para publicação em 19 de maio de 2003

Recebido para publicação, após modificações, em 4 de fevereiro de 2004

E-mail: dete.s@via-rs.net 
Como a LDL é a lipoproteína responsável pelo transporte do colesterol para os tecidos, o aumento de seus níveis leva ao acúmulo no plasma e conseqüente aderência ao endotélio vascular. Com isso, há a formação de um núcleo, gerador de um trombo que pode ocasionar infarto. A deposição de colesterol nos vasos leva ao enrijecimento, espessamento e diminuição da luz arterial, que é chamado de ateroma e ocorre quando as LDLs atravessam os endotélios e são oxidadas, o que altera sua estrutura original (Berne e Levy, 1998). A partir daí, essas lipoproteínas passam a ser consideradas corpos estranhos e são fagocitadas por macrófagos, constituindo as células em espuma que, ao se acumularem, diminuem o diâmetro dos vasos por espessamento que ocorre da região serosa em direção à luz vascular, ocasionando a chamada aterosclerose (Berne e Levy, 1998; Riegel, 2001). Devido a essas características, a LDL é chamada de "mau colesterol".

A função de transporte reverso do colesterol realizado pela HDL confere a essas partículas o nome de "bom colesterol", pois elas contribuem de maneira significativa para a manutenção dos níveis de lipoproteínas fisiologicamente apropriados e saudáveis, ajudando assim a prevenir a aterosclerose por facilitar a captação celular de LDL (Berne e Levy, 1998).

Em um experimento realizado com 362 cães, 53 deles apresentaram valores elevados de lipoproteínas. Desses, $81 \%$ foram atribuídos a distúrbios metabólicos, tais como hipotireoidismo, diabete melito, hiperadrenocorticismo, doenças renais e hepáticas (Watson e Barrie, 1993).

Em cães a aterosclerose não é um problema freqüente. De 21 casos encontrados na necropsia, durante os anos de 1970 a 1983, 18 animais eram machos e três fêmeas, duas delas castradas (Liu et al., 1986).

Em um levantamento sobre os valores das lipoproteínas em diferentes raças de cães saudáveis, foram encontradas diferenças significativas nos valores de triglicerídeos (TG), LDL e HDL dos animais em relação aos valores fisiológicos no homem. Os valores de HDL foram mais elevados, $65 \%$ a mais do colesterol total na maioria das raças e $82 \%$ a mais nos Cairn Terriers (Downs, 1993).
Em cães, gatos, cavalos, ruminantes e camundongos predomina a fração HDL, o que poderia justificar a resistência dessas espécies ao surgimento do ateroma espontâneo (Bauer, 1996).

Em mulheres, a reposição hormonal após a menopausa pode melhorar significativamente o perfil lipoprotéico (Giannini, 2001), não pela administração de progesterona, que não possui efeito significativo na redução das lipoproteínas (Mukherja e Biswas, 1975), e sim pelo uso do estrógeno, que reduz em até $50 \%$ os riscos de doenças coronarianas pela redução do colesterol total e da LDL e elevação da HDL (Giannini, 2001).

Os estrógenos suprimem a atividade da lipase hepática (LH), enzima que possui efeito de degradação da HDL. Com isso, quanto menor o nível estrogênico, maior a atividade da $\mathrm{LH}$ e menor a concentração de HDL. Além disso, os estrógenos parecem elevar a HDL pela elevação na síntese da apoproteína AI (Tikkanen e Nikkila, 1987).

Os valores de colesterol e TG são obtidos no soro ou plasma por meio de testes enzimáticos de fácil execução e baixo custo ( Barrie et al., 1993; Watson e Barrie, 1993), sendo necessário jejum de 12 (Zerbe, 1988; Downs et al., 1994 ) a 16 horas (Johnson, 1992; Kaneko, 1997).

Este trabalho teve como objetivo avaliar a influência da ovário-histerectomia e da reposição com estrógenos no perfil lipoprotéico de cadelas.

\section{MATERIAL E MÉTODOS}

Foram utilizadas 12 cadelas adultas, entre dois e seis anos, pesando entre 5 e $15 \mathrm{~kg}$. Durante o período de adaptação, com duração de 60 dias, os animais receberam vermífugo à base de febantel, praziquantel e pamoato de pirantel ${ }^{1}$ na dose de 1 comprimido para $10 \mathrm{~kg}$ de peso. Foram vacinados contra cinomose, leptospirose, hepatite infecciosa canina e parvovirose $\mathrm{e}^{2}$ e receberam um agente tópico pulicida e carrapaticida (fipronil) ${ }^{3}$.

\footnotetext{
${ }^{1}$ DRONTAL PLUS. Bayer S.A. Rua Domingos Jorge, 1100

- Socorro, SP

${ }^{2}$ BIOVAC SIX. LEMA BIOLOGICAL do Brasil

${ }^{3}$ FRONTLINE. Merial Saúde Animal LTDA. Fazenda São

Francisco - Paulínia, SP
} 
Os cães foram mantidos com água ad libitum e ração comercial ${ }^{4}$, constituída de $19 \%$ de proteína bruta, $12 \%$ de umidade, $48 \%$ de glicídios, $4 \%$ de matéria fibrosa, $5 \%$ de lipídeos e $12 \%$ de matéria mineral, sendo $2 \%$ de fósforo e $1,5 \%$ de cálcio, equivalente a um valor nutricional de $3.300 \mathrm{kcal} / \mathrm{kg}$ de alimento. A ração foi fornecida conforme o peso do grupo, duas vezes ao dia, com base em tabela nutricional referenciada por Kirk (1988).

Após a fase de adaptação, as fêmeas, submetidas à ovario-histerectomia, foram distribuídas em dois grupos de seis animais. Um dos grupos, denominado reposição hormonal $(\mathrm{RH})$, recebeu, via oral, $0,01 \mathrm{mg} / \mathrm{kg}$ de estrógenos naturais conjugados $^{5}$, diluídos em azeite de oliva, a cada 48 horas, durante 12 meses. O outro grupo, controle (C), recebeu apenas o azeite de oliva como placebo.

No dia da castração, momento 1 (M1), e a cada 60 dias (M2, M3, M4, M5 e M6) foram realizadas colheitas de sangue e feitos exames de dosagem de colesterol total, triglicerídios, HDL, LDL e VLDL. O soro congelado de cada duas colheitas foi processado e analisado no mesmo dia.

Como controle de qualidade desses ensaios foram realizados os seguintes procedimentos: estabelecimento de uma curva-padrão como referência, com diluições seriadas contendo de $25 \mathrm{mg} \%$ a $250 \mathrm{mg} \%$; utilização de "pools" para cálculo de variação intra e interensaio e determinação dos valores de cada amostra em duplicata.

A determinação dos níveis séricos do colesterol total, triglicerídios e HDL foi feita por espectofotometria, pelo método enzimáticocolorimétrico Bioclin 6 , com a utilização de "kits" comerciais Bioclin-Colesterol-Cod-Clin, Bioclin triglicérides enzimático e Bioclin colesterol HDL enzimático, respectivamente. Os valores foram expressos à $37^{\circ} \mathrm{C}, \mathrm{em} \mathrm{mg} / \mathrm{dl}$.

\footnotetext{
${ }^{4}$ PEDIGREE MARROWBONE. Div. Éffem Produtos Alimentícios. Br 116, Km 286, Eldorado do Sul, RS ${ }_{5}^{5}$ PREMARIM. Laboratórios WYETH. Rua Alexandre Dumas 2200 - São Paulo, SP

${ }^{6}$ BIOCLIN. Quibasa. Rua Teles Menezes, 92, Belo

Horizonte, MG
}

A concentração das lipoproteínas de muito baixa densidade foi calculada segundo a equação de Friedwald: $\mathrm{VLDL}=\mathrm{TG} \div 5$. Os valores foram expressos em $\mathrm{mg} / \mathrm{dl}$ e usados para o cálculo da concentração de LDL.

Os níveis séricos das lipoproteínas de baixa densidade foram obtidos também segundo a equação de Friedwald, após a obtenção dos valores de colesterol total, triglicerídeos, HDL e VLDL: LDL = C-[HDL + VLDL], expressos em $\mathrm{mg} / \mathrm{dl}$.

A metodologia estatística utilizou a análise de variância para a comparação entre os grupos e o teste de Tukey para a comparação entre os momentos. O nível de significância considerado foi $\mathrm{P}<0,05$.

\section{RESULTADOS E DISCUSSÃO}

Os valores de colesterol total obtidos em M1 estão dentro dos níveis normais para a espécie, cuja variação, segundo Kaneko (1997), é de 130 a $270 \mathrm{mg} / \mathrm{dl}$. A partir de M2, houve elevação nos valores do colesterol total no grupo $\mathrm{C}(\mathrm{P}=0,023)$ e manutenção ou declínio deles no grupo $\mathrm{RH}$ (Fig. 1).

Estes resultados são compatíveis com os citados na literatura humana, em que mulheres, após a menopausa ou gonadectomia, apresentam elevação nos níveis de colesterol total. Após o início da reposição estrogênica, os valores tendem a diminuir, permanecendo dentro da normalidade (Wolfe e Huff, 1995; Speroff et al., 1996; Sullivan, 1996; Giannini, 2001).

Os valores do colesterol total apresentaram pequena redução após o início da reposição hormonal, variando apenas de 5 a $10 \%$, pois o que realmente é passível de efeito estrogênico são as frações HDL e LDL (Wolfe e Huff, 1995). 


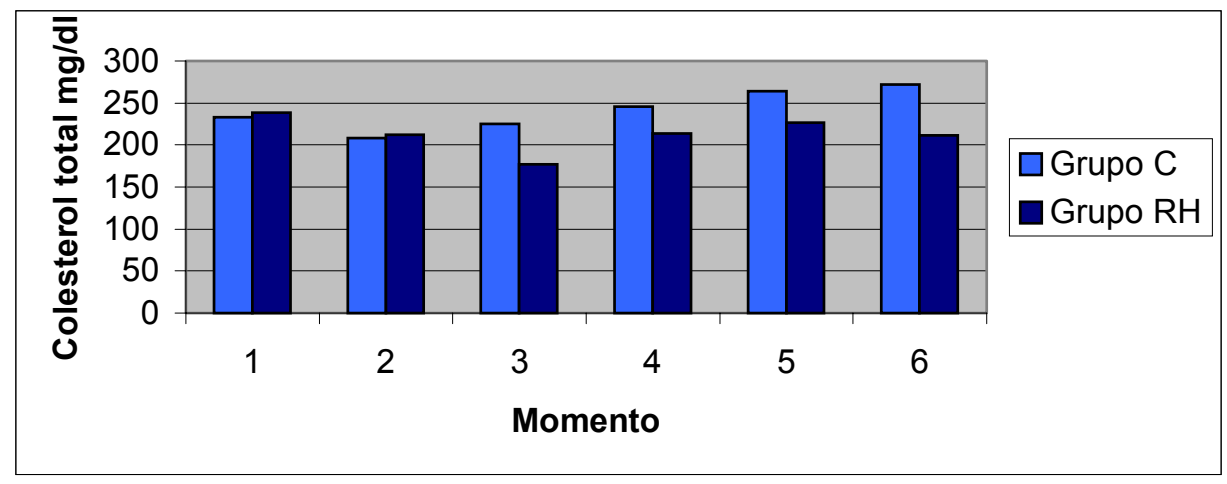

Figura 1. Médias do colesterol total, expressas em mg/dl, em animais dos grupos controle (C) e de reposição hormonal $(\mathrm{RH})$, durante os seis momentos.

Algumas fêmeas do grupo $\mathrm{C}$ apresentaram elevação da colesterolemia já em M2, que se tornou mais significativa a partir de M3, conferindo com os dados de experimentos em mulheres, nos quais o aumento do colesterol em situações de privação estrogênica ocorreu de forma rápida (Giannini, 2001). Esse aumento pode ou não ser progressivo e varia individualmente, havendo casos de elevação muito acima dos limites considerados normais, o que predispõe as mulheres às doenças coronarianas (Speroff et al., 1996). No grupo C, quatro das seis fêmeas apresentaram em M6 valores acima do limite normal máximo. Nos animais do grupo RH, todos os valores permaneceram dentro da normalidade.

Em ratas submetidas à ovariectomia, a reposição com estrógenos causou decréscimo no conteúdo dos lipídeos totais em todos os tecidos, indicando seu efeito benéfico, cuja ação ocorre não somente na quantidade de lipídeos circulantes mas também nos tecidos de maneira geral (Mukherja e Biswas, 1975).

Os níveis de triglicerídeos (Fig. 2) diminuíram discretamente em M2, seguindo-se pequena elevação nos dois últimos momentos no grupo $\mathrm{C}$ $(\mathrm{P}=0,041)$, oscilações que permaneceram dentro dos limites físiológicos para a espécie, cujos valores variam de 80 a $150 \mathrm{mg} / \mathrm{dl}$ (Kaneko, 1997). No grupo RH, as médias de triglicerídeos permaneceram próximas da média em M1 e apresentaram leve declínio em M5 e M6 $(\mathrm{P}=$ 0,067).

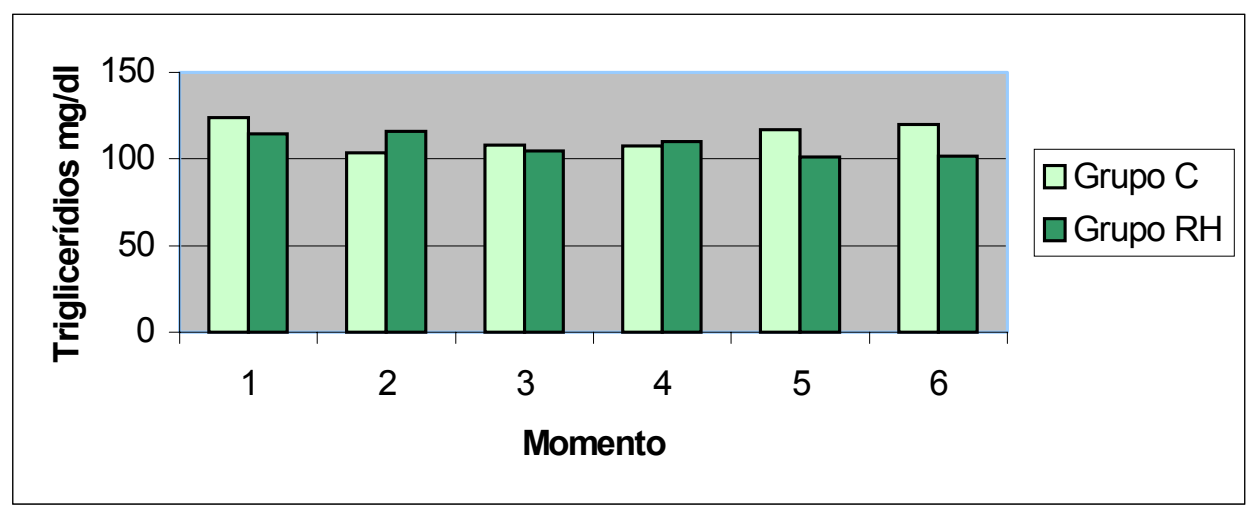

Figura 2. Médias dos triglicerídeos, expressas em $\mathrm{mg} / \mathrm{dl}$, em animais dos grupos controle (C) e de reposição hormonal $(\mathrm{RH})$, durante os seis momentos. 
Em mulheres, existe uma tendência geral para a elevação dos níveis de triglicerídeos com a reposição hormonal (Speroff et al., 1996), o que pode ser atribuído à administração oral de estrogênios, que causa aumento na produção de VLDL, ocasionando, com isso, acréscimo nos valores de triglicerídeos (Basdevant 1992). Esse parece ser o único efeito indesejado no perfil lipoprotéico de mulheres que receberam estrógenos pós-menopausa (Sullivan, 1996) e que não pôde ser constatado no presente experimento.

A lipoproteína HDL tende a ser a fração predominante no cão, representando em torno de $65 \%$ do colesterol total na maioria das raças
(Crispin et al., 1992; Downs, 1993, Bauer, 1996). Isso não pôde ser constatado neste estudo, no qual os níveis de HDL em M1, considerados como valores basais, variaram de 34 a $50 \%$ do colesterol total, com predominância da fração LDL, e variação de 37 a 58\% nos valores da concentração total de colesterol.

As médias de HDL nos diferentes momentos (Fig. 3) indicam mudanças no grupo C. Os níveis de HDL diminuíram já a partir de M2, até M6. A redução foi de, aproximadamente, $45 \%$ em M6 $(\mathrm{P}=0,015)$. No grupo $\mathrm{RH}$, os valores foram semelhantes de M1 a M6.

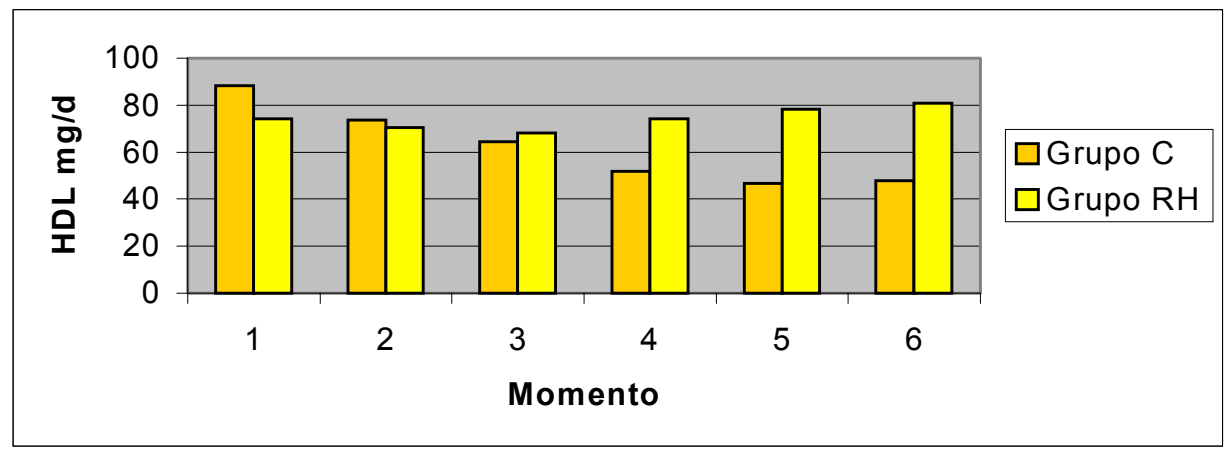

Figura 3. Médias das HDL, expressas em mg/dl, em animais dos grupos controle (C) e de reposição hormonal $(\mathrm{RH})$, durante os seis momentos.

Valores normais de HDL (entre 45 e $60 \%$ do colesterol total) foram encontrados em M1 para ambos os grupos. A redução observada no grupo $\mathrm{C}$ revela comportamento similar ao perfil lipoprotéico de mulheres na menopausa, as quais apresentam tendência progressiva à diminuição da fração HDL após a baixa dos níveis estrogênicos (Wolfe e Huff, 1995; Speroff et al., 1996; Sullivan 1996).

No grupo RH, houve elevação do HDL em alguns animais ou não se alterou em outros, indicando a possibilidade de manutenção desses níveis por meio da reposição hormonal.

Relatos de experimentos em mulheres citam a elevação nos níveis de HDL após o início da reposição hormonal. A menopausa surge em torno dos 48 anos, com evolução aproximada de
44 meses, ou seja, o quadro se instala lenta e progressivamente, permitindo, com isso, decréscimo nos valores dessa lipoproteína pela falta estrogênica (Castañeda e Pachón, 2001). Os níveis da fração HDL retornam à normalidade após a reposição hormonal, aspecto confirmado neste estudo, isto é, não houve acréscimo significativo de HDL no grupo que recebeu estrógeno porque não chegou a haver decréscimo. (Giannini, 2001),

A ação específica dos esteróides estrogênicos parece estar relacionada à atividade da enzima lipase hepática (Basdevant, 1992), cuja função é degradar os fosfolipídeos e os triglicerídeos da HDL, ou seja, níveis adequados de estrógenos suprimem fisiologicamente a atividade da lipase hepática, elevando os valores principalmente de HDL2. Além disso, a síntese de apolipoproteína 
AI também está aumentada durante a reposição hormonal, o que favorece a formação de HDL (Tikkanen e Nikkila, 1987).

Os níveis de LDL (Fig. 4) apresentaram elevação crescente nas fêmeas do grupo $\mathrm{C}$, já perceptível em alguns animais em M2 e, em outros, a partir de M3. Na última dosagem, todos os animais apresentavam valores bem acima dos basais, cujos valores variam entre 40 e $55 \%$ do colesterol total $(\mathrm{P}=0,012)$.

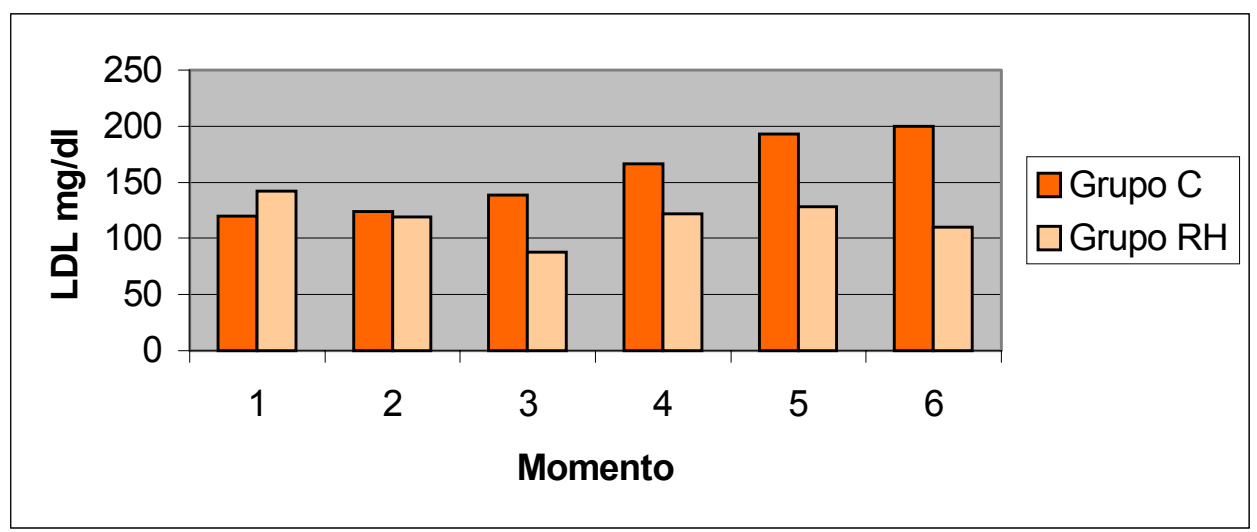

Figura 4. Médias das LDL, expressas em mg/dl, em animais dos grupos controle (C) e de reposição hormonal (RH), durante os seis momentos.

No grupo RH, a fração LDL oscilou nos três primeiros momentos e, a partir de M4, diminuiu. Em M6, o valor foi menor que em M1 (P= 0,023).

Estes dados confirmam os encontrados na literatura, que cita como um dos principais efeitos da reposição estrogênica a manutenção ou a diminuição dos níveis de LDL em mulheres na menopausa. A falta de LDL causa elevação desses valores, favorecendo o surgimento de coronariopatias (Wolfe e Huff, 1995; Speroff et al., 1996; Sullivan, 1996; Castañeda e Pachón, 2001).

Em cães, é raro o surgimento de aterosclerose. O diagnóstico geralmente é feito durante a necropsia, e os relatos de casos citam prevalência maior em machos (Liu et al., 1986), que não têm a mesma proteção relativa das fêmeas, atribuída aos níveis estrogênicos mais elevados (Berne e Levy, 1998).

Os machos tendem a apresentar níveis de LDL mais elevados durante toda a vida. As fêmeas, após a castração, perdem a vantagem do efeito estrogênico, passando a fazer parte do grupo de maior risco para a ateroesclerose (Joossens, 1988). Dos casos relatados em fêmeas, $75 \%$ eram castradas e não apresentavam doença endócrina concomitante (Liu et al., 1986).

A fração LDL em mulheres tende a diminuir, em torno de 15 a $20 \%$, após o início da reposição estrogênica (Berne e Levy, 1998), aspecto confirmado no presente experimento, cuja redução foi, em média, $20 \%$.

Além do efeito benéfico sobre as lipoproteínas, a reposição estrogênica tende a restaurar a produção de óxido nítrico, baixar o nível sérico de fibrinogênio, diminuir a oxidação das LDL e aumentar a produção de prostaciclinas pelo endotélio (McHugh et al., 1998), diminuindo, com isso, os riscos de doenças coronarianas.

As médias de VLDL (Fig. 5) indicam que houve oscilação nos níveis dessa lipoproteína tanto no grupo C, cujos valores em M6 eram semelhantes aos valores basais (aproximadamente 5\% do colesterol total), quanto no grupo $\mathrm{RH}$, que, no final do estudo, estavam levemente mais baixos do que em M1. Esses valores permaneceram dentro dos limites fisiológicos durante todo o período experimental $(\mathrm{P}=0,082)$. 


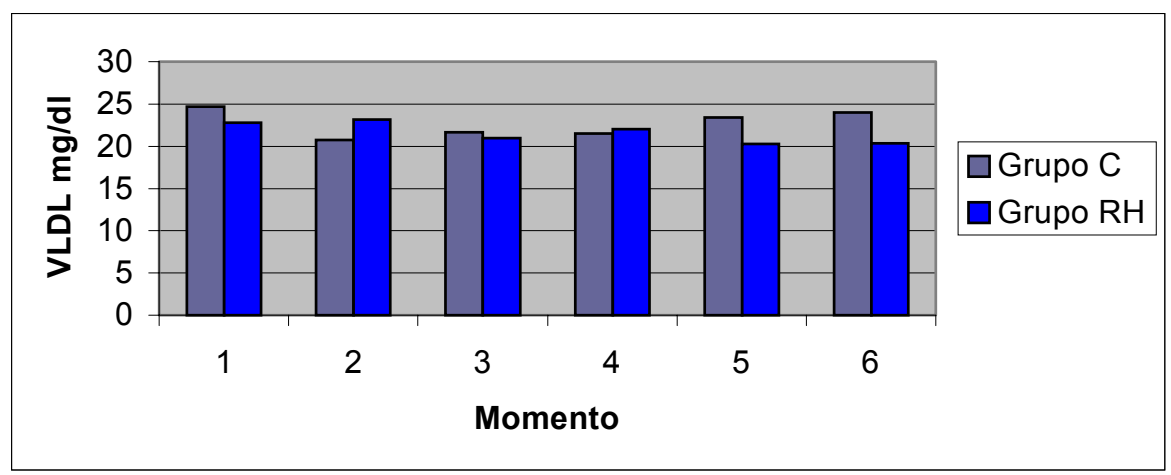

Figura 5. Médias das VLDL, expressas em mg/dl, nos animais dos grupos controle (C) e de reposição hormonal (RH) durante os seis momentos.

A reposição estrogênica em mulheres tende a elevar de forma branda a produção de triglicerídeos e, conseqüentemente, de VLDL (Wolfe e Huff, 1995). No presente experimento, não houve elevação nos níveis de triglicerídeos $\mathrm{e}$ de VLDL no grupo que recebeu hormônio.

\section{CONCLUSÕES}

A partir dos resultados expressos nesse trabalho é possível concluir que: a ovário-histerectomia influi no perfil lipoprotéico, promovendo elevação do colesterol total e da fração LDL e a reposição estrogênica é capaz de manter os valores do perfil lipoprotéico de cadelas submetidas à ovário-histerectomia.

\section{REFERÊNCIAS BIBLIOGRÁFICAS}

BARRIE, J.; WATSON, T.D.G.; STEAR, M.J. et al. Plasma cholesterol and lipoprotein concentrations in the dog: the effects of age, breed, gender and endocrine disease. J. Small Anim. Pract., v.34, p.507-512, 1993.

BASDEVANT, A. Steroids and lipid metabolism: mechanism of action. Int. J. Fertil., v.37, p.93-97, 1992.

BAUER, J.E. Comparative lipid and lipoprotein metabolism. Vet. Clin. Pathol., v.25, p.49-56, 1996.

BERNE, M.R.; LEVY, M.N. Fisiologia. 4.ed. Rio de Janeiro: Guanabara Koogan, 1998. 1034p.
CASTAÑEDA, G.B.; PACHÓN, J.A.P. Efecto de la terapia estrogenica y combinada estrogenoprogestacional sobre el perfil lipidico en pacientes com suplencia para sindrome climaterico. [on line] Disponível em: $<$ http://www.encolombia.com/efectos-menos$1 \mathrm{htm}>$. Acessado em: 29 jun.2001.

CRISPIN, S.M.; BOLTON, C.H.; DOWNS, L.G. Plasma lipid and lipoprotein profile of working and pet border collies. Vet. Rec., v.130, p.185186, 1992.

DOWNS, L.G. Plasma lipoprotein lipids in five different breeds of dogs. Res. Vet. Sci., v.54, p.63-67, 1993.

DOWNS, L.G.; WILLS, J.M.; ZANI, V. et al. Changes in plasma lipoprotein during the oestrus cycle of the bitch. Res. Vet. Sci., v.56, p.82-88, 1994.

GIANNINI, S.D. Lipoproteínas como fatores de risco em mulheres. Rev. Soc. Cardiol. Estado São Paulo. V.6 Disponível em: $<$ http://www.socesp.org.br/revistas/v6n6/htm>. Acessado em: 15 fev. 2001

JOHNSON, K.R. Hiperlipidemia canina. In: ETTINGER, S.J. Tratado de medicina interna veterinária. 3.ed. São Paulo: Manole, 1992. V.1, p.210-215.

JOOSENS, J.V. Mechanisms of hypercholesterolemia and atherosclerosis. Acta Cardiol. Suppl., v.29, p.63-83, 1988.

KANEKO, J.J. Lipids and ketones. In: CLINICAL biochemistry of domestic animals. 5.ed. San Diego: Academic, 1997. p.83-115. 
KIRK, R.W. Atualização terapêutica veterinária. 2.ed. São Paulo: Manole, 1988. V.2, p.13191329.

LIU, S.; TILLEY, L.P.; TAPPE, J.P. et al. Clinical and pathological findings in dogs with atherosclerosis: 21 cases. J. Am.Vet. Med. Assoc., v.189, p.227-232, 1986.

McHUGH, N.A.; SOLOWIEJ, A.; STERNBERG, L. et al. Cardiac and coronary vascular effects of chronically adminestered estrogen in the dog. Basic Res. Cardiol., v.93, p.116-121, 1998.

MUKHERJA, M.; BISWAS, $\mathrm{R}$ Effect of ovariectomy and replacement therapy on the tissue lipid pattern in rats. Endokrinologie, v.66, p.113-121, 1975.

RIEGEL, E.R. Bioquímica. 3.ed. São Leopoldo: Unisinos, 2001. 547p.

SPEROFF, L.; ROWAN, J.; SYMONS, J. et al. The comparative effect on bone density, endometrium, and lipids of continuous hormones as replacement therapy. J. Am. Med. Assoc., v.6, p.1397-1403, 1996

SULLIVAN, J.M. Estrogen replacement therapy. Am. J. Med., v.101, p.56S-60S, 1996.

TIKKANEN, M.J.; NIKKILÄ, E.A., Regulation of hepatic lipase and serum lipoproteins by Sex steroids. Am. Heart J., v.113, p.562-567, 1987.

WATSON, T.D.G.; BARRIE, J. Lipoprotein metabolism and hyperlipidaemia in the dog and cat: a review. J. Small Anim. Pract., v.34, p.479$487,1993$.

WOLFE, B.M.; HUFF, M.W. Effects of continuous low-dosage hormonal replacement therapy on lipoprotein metabolism in postmenopausal women. Metabolism, v.44, p.410-417, 1995.

ZERBE, C.A. Hiperlipemias caninas. In: KIRK, R.W. Atualização terapêutica veterinária. 2.ed. São Paulo: Manole, 1988. v.2, p.1319-1329. 